\title{
A eficácia do mentol como anestésico para tambaqui (Colossoma macropomum, Characiformes: Characidae).
}

\author{
Michelle Ferreira FAÇANHA ${ }^{1}$ e Levy de Carvalho GOMES ${ }^{2}$
}

\begin{abstract}
RESUMO
Os anestésicos são importantes na piscicultura para reduzir o estresse e a mortalidade no manejo. Este trabalho tem como objetivo determinar a eficácia do mentol para tambaqui durante o manejo. Na primeira série de testes, foi examinado o efeito da concentração de anestésico sobre indução à anestesia e o estresse de tambaqui. Na segunda série de testes, foi avaliada a recuperação dos peixes após a exposição a uma concentração de $150 \mathrm{mg} / \mathrm{L}$ de mentol por diferentes tempos. Na terceira série, foi avaliada se a melhor concentração encontrada para juvenil $(150 \mathrm{mg} / \mathrm{L})$ também era adequada para peixes maiores. A melhor concentração para uma anestesia cirúrgica foi $150 \mathrm{mg} / \mathrm{L}$, pois o tempo de indução é rápido, porém a recuperação é significativamente mais demorada do que para as menores concentrações testadas. Para uma anestesia, com finalidade de biometria, a melhor concentração foi $100 \mathrm{mg} / \mathrm{L}$. Nesta concentração o tempo de indução à anestesia é prolongado, porém o tempo de recuperação está dentro da faixa considerada adequada. O tempo de recuperação do tambaqui quando exposto a $150 \mathrm{mg} / \mathrm{L}$ é significativamente igual para 10, 20 e 30 minutos de anestesia. Os resultados obtidos mostram que o mentol é um anestésico eficiente para o tambaqui.
\end{abstract}

PALAVRAS-CHAVE

anestesia, sedação, peixe, manejo, estresse.

\section{Efficacy of mentbol as an anestbetic for tambaqui (Colossoma macropomum, Characiformes: Characidae).}

\begin{abstract}
Anesthetics are important in fish culture to reduce handling stress and mortality. The objective of this work is to investigate menthol as an anesthetic for tambaqui. In the first series of tests, fish were exposed to various concentrations of menthol to evaluate induction time and stress responses. The second series examined the effect of exposure period to menthol at $150 \mathrm{mg} / \mathrm{L} \mathrm{on}$ recovery time. The third assessed the best dosage for juveniles in larger tambaqui. The best concentration for surgical anesthesia is $150 \mathrm{mg} / \mathrm{L}$. At this concentration the induction time is short, but their recovery time is significantly longer than that for lower concentrations. For biometry procedures, the best concentration is $100 \mathrm{mg} / \mathrm{L}$. At this concentration the induction time is prolonged, but the recovery time is within the desired period. Recovery time for fish exposed to $150 \mathrm{mg} / \mathrm{L}$ is equal for 10,20 or 30 minutes of exposure. The results confirmed that menthol is an adequate anesthetic for tambaqui.
\end{abstract}

\section{KEYWORDS}

anesthesia, sedation, fish, handling, stress.

\section{INTRODUÇÃO}

Anestésicos são importantes na piscicultura para reduzir o estresse e a mortalidade no manejo. Vários produtos químicos são usados para anestesia de peixes, sendo os mais comuns a tricaína metanosulfato (MS222), a quinaldina e o 2-fenoxietanol (Mgbenka \& Ejiofor, 1998; Ross \& Ross, 1999; Hovda \& Linley, 2000). Porém, estes anestésicos são de difícil obtenção e apresentam alto custo (Roubach \& Gomes, 2001).
Não existem leis que regulamentem o uso de anestésicos para peixes no Brasil. Desta forma, procura-se seguir as recomendações da Food and Drug Administration (FDA). A obtenção de anestésicos é difícil, pois o MS222, o único anestésico químico aprovado pelo FDA para uso em peixes, não é produzido no Brasil. O seu preço é proibitivo, sendo a concentração necessária para anestesiar um peixe cerca de dez vezes mais cara que uma concentração similar de benzocaína

${ }^{1}$ Escola Superior Batista do Amazonas. Endereço atual: INPA/CPAQ, Caixa Postal 478, CEP 69011-970, Manaus, AM.

${ }^{2}$ Embrapa Amazônia Ocidental, Caixa Postal 319, CEP 69011-970, Manaus, AM. E-mail: levy@cpaa.embrapa.br. Autor para correspondência. 
(Gomes et al., 2001). Desta forma, justifica-se a necessidade de se buscar alternativas seguras para procedimentos de anestesia de peixes no Brasil, subsidiando as autoridades responsáveis por este tipo de regulamentação.

$\mathrm{O}$ anestésico mais utilizado no Brasil é a benzocaína. Resultados obtidos por Gomes et al. (2001) com juvenis de tambaqui sugerem que a benzocaína atende a maioria dos critérios estabelecidos por Ross \& Ross (1999) para um anestésico ideal para peixes. De forma similar à benzocaina, $\mathrm{o}$ mentol tem várias características que o qualificam como um anestésico adequado para peixes: eficácia e boa margem de segurança para o peixe e para o operador na concnetração utilizada (Roubach \& Gomes, 2001).

O mentol é um óleo essencial extraído de plantas do gênero Mentha (Martins et al., 2000), com conhecida propriedade anestésica (Ruppert \& Barnes, 1994) e antiinflamatória (Lorenzo et al., 2002). Uma vantagem do uso do mentol como anestésico para peixes é que este é utilizado em farmácias de manipulação, sendo a exemplo da benzocaína, facilmente encontrado no mercado local a baixo custo.

O objetivo deste trabalho foi determinar a eficácia do mentol durante o manejo e determinar um protocolo de anestesia com este produto para tambaqui. Para isto, foram avaliadas várias concentrações para anestesia e o tempo máximo de exposição à solução de mentol.

\section{MATERIAL E MÉTODOS}

Juvenis de tambaqui foram obtidos na Estação de Piscicultura de Balbina, Amazonas-Brasil. Os peixes $(n=74,88,69 \pm 23,85 \mathrm{~g}$; média \pm desvio padrão) foram aclimatados em aquários de 120 L, com sistema de aeração constante, por 7 dias.

Os testes foram conduzidos em aquários estáticos de $6 \mathrm{~L}$, contendo apenas $3 \mathrm{~L}$ de água. O mentol foi dissolvido em uma concentração de $1 \mathrm{~g} / 10 \mathrm{~mL}$ de álcool para o preparo da solução estoque (100 mg de mentol para cada mL de solução). A água do aquário foi trocada ao término de cada teste. Os protocolos experimentais estavam de acordo com Waterstrat (1999) e Gomes et al. (2001).

Na primeira série de testes, foi examinado o efeito da concentração de mentol sobre à indução a anestesia e o estresse de tambaqui. Os peixes $(n=10$ para cada concentração) foram individualmente expostos a concentrações de 50, 100, 150, 200 e $250 \mathrm{mg} / \mathrm{L}$ de mentol por dez minutos. Durante a exposição, foram observados os diferentes estágios de indução à anestesia. Após este período, os peixes foram removidos da solução de anestésico e colocados em um aquário com capacidade de $60 \mathrm{~L}$, contendo $40 \mathrm{~L}$ de água e aeração constante, para monitorar a recuperação. Imediatamente após a recuperação, foi coletado sangue dos vasos caudais de 5 peixes de cada concentração. Com o sangue foram analisados a glicemia e os íons plasmáticos $\mathrm{Na}^{+}$e $\mathrm{K}^{+}$. A glicose foi mensurada com um leitor digital (Advantage ${ }^{\mathrm{TM}}$ ) e os íons analisados em um fotômetro de chama.
Na segunda série de testes, foi avaliado o efeito de duração da anestesia no tempo de recuperação do tambaqui. Os peixes $(n=8$ para cada tempo de exposição) foram expostos individualmente à concentração de $150 \mathrm{mg} / \mathrm{L}$ de mentol por 10,20 e 30 minutos e posteriormente transferidos para recuperação em um aquário de $60 \mathrm{~L}$ contendo $40 \mathrm{~L}$ de água com aeração constante. Após a recuperação os peixes foram transferidos para um aquário de criação contendo $120 \mathrm{~L}$ pelo período de 96 horas para monitorar a mortalidade.

Na terceira série de testes, a concentração mais adequada para juvenis de $88 \mathrm{~g}(150 \mathrm{mg} / \mathrm{L})$ foi testada em peixes maiores ( $415 \pm 20,33 \mathrm{~g}$; média \pm desvio padrão). Os peixes $(n=5)$ foram expostos individualmente à concentração de $150 \mathrm{mg} / \mathrm{L}$ de mentol em um aquário de $60 \mathrm{~L}$ com $25 \mathrm{~L}$ de água, durante dez minutos, quando foram observados os diferentes estágios de indução à anestesia. Após a exposição ao anestésico, os peixes foram colocados em um aquário de $60 \mathrm{~L}$, contendo $40 \mathrm{~L}$ de água e aeração constante, para monitorar a recuperação.

Para avaliar os estágios de evolução da anestesia foi usado critério proposto por Stoskopf (1993). O peixe foi considerado recuperado quando retornava os movimentos e nadava ativamente (Gomes et al., 2001). O tempo para atingir os diferentes estágios de anestesia e a recuperação, assim como, a glicose e os íons plasmáticos, foram avaliados para as diferentes concentrações de mentol por uma análise de variância e teste de Tukey a 5\% de probabilidade. Os diferentes estágios de anestesia foram comparados entre juvenis e os peixes maiores pelo teste $\mathrm{T}$ de Student a 5\% de probabilidade. As análises foram realizadas pelo pacote estatístico Systat 9.0.

\section{RESULTADOS E DISCUSSÃO}

O tambaqui exposto ao mentol (50-250 mg/L) passa seqüencialmente por todos estágios de anestesia descritos por Stoskopf (1993) (Tabela 1). O tempo para indução à perda de reação a estímulos é semelhante para todas as concentrações testadas. O tempo para perda parcial e total de equilíbrio, redução e parada dos movimentos operculares é semelhante nos peixes expostos a 50 e $100 \mathrm{mg} / \mathrm{L}$ e significativamente maior que nos peixes expostos às demais concentrações. O tempo para indução à perda total de equilíbrio e parada dos batimentos operculares dos peixes anestesiados com mentol é significativamente maior na concentração $100 \mathrm{mg} / \mathrm{L}$ do que $150 \mathrm{mg} / \mathrm{L}$. A concentração 150 $\mathrm{mg} / \mathrm{L}$ de mentol é a mais adequada para indução de anestesia cirúrgica, pois o tempo de indução a perda total de equilíbrio é de 0,51 minutos e o da concentração $100 \mathrm{mg} / L$ é de 1,92 minutos. Para a parada total dos batimentos operculares, o tempo de indução à anestesia, quando exposto a uma concentração de $150 \mathrm{mg} / \mathrm{L}$ é significativamente menor (2,16 minutos) do que quando exposto a $100 \mathrm{mg} / \mathrm{L}$ (4,39 minutos). Resultado semelhante foi obtido por Roubach et al. (2001) para anestesia de matrinxã (Brycon cephalus) com 150 mg/L MS222. Porém, uma concentração de MS222 pouco superior a ideal causa mortalidade para o matrinxã, enquanto o mentol apresenta uma boa margem de segurança para o tambaqui, pois não ocorreu mortalidade entre os peixes nas concentrações testadas. 


\section{ACTA AMAZONICA}

Tabela 1 - Eventos comportamentais dos juvenis de tambaqui expostos a diferentes concentrações de mentol. Os estágios de anestesia estão de acordo com os critérios de Stoskopf (1993). Recuperação = recuperação do equilíbrio e natação ativa. Médias seguidas pela mesma letra em coluna não diferem entre si pelo teste de Tukey (5\%); os resultados são média \pm desvio padrão.

\begin{tabular}{lcccccc}
\hline \hline \multicolumn{5}{c}{ Eventos comportamentais (minutos) } & \\
\hline $\begin{array}{l}\text { Mentol } \\
\text { (mg/L) }\end{array}$ & $\begin{array}{c}\text { Perdade reação } \\
\text { à estímulos }\end{array}$ & $\begin{array}{c}\text { Perda parcial } \\
\text { de equilíbrio }\end{array}$ & $\begin{array}{c}\text { Perda total } \\
\text { de equilíbrio }\end{array}$ & $\begin{array}{c}\text { Redução dos } \\
\text { batimentos operculares batimentos operculares }\end{array}$ & \multicolumn{2}{c}{ Parada dos } \\
\hline 50 & $0,24 \pm 0,03^{\mathrm{a}}$ & $1,08 \pm 0,14^{\mathrm{a}}$ & $2,58 \pm 0,29^{\mathrm{a}}$ & $3,36 \pm 0,40^{\mathrm{a}}$ & $5,97 \pm 0,39^{\mathrm{a}}$ & $5,32 \pm 0,55^{\mathrm{a}}$ \\
100 & $0,29 \pm 0,05^{\mathrm{a}}$ & $1,22 \pm 0,14^{\mathrm{a}}$ & $1,92 \pm 0,25^{\mathrm{a}}$ & $2,59 \pm 0,26^{\mathrm{ab}}$ & $4,39 \pm 0,42^{\mathrm{b}}$ & $5,04 \pm 0,66^{\mathrm{a}}$ \\
150 & $0,17 \pm 0,02^{\mathrm{a}}$ & $0,29 \pm 0,02^{\mathrm{b}}$ & $0,51 \pm 0,11^{\mathrm{b}}$ & $1,50 \pm 0,29^{\mathrm{bc}}$ & $2,16 \pm 0,34^{\mathrm{c}}$ & $10,93 \pm 1,72^{\mathrm{b}}$ \\
200 & $0,18 \pm 0,02^{\mathrm{a}}$ & $0,39 \pm 0,09^{\mathrm{b}}$ & $0,96 \pm 0,17^{\mathrm{b}}$ & $1,37 \pm 0,19^{\mathrm{c}}$ & $2,03 \pm 0,28^{\mathrm{c}}$ & $12,54 \pm 2,11^{\mathrm{b}}$ \\
250 & $0,21 \pm 0,04^{\mathrm{a}}$ & $0,33 \pm 0,04^{\mathrm{b}}$ & $0,72 \pm 0,15^{\mathrm{b}}$ & $1,53 \pm 0,27^{\mathrm{bc}}$ & $1,76 \pm 0,30^{\mathrm{c}}$ & $12,94 \pm 1,53^{\mathrm{b}}$ \\
\hline \hline
\end{tabular}

Para anestesia voltada para biometria e breve manejo, a concentração recomendada é $100 \mathrm{mg} / \mathrm{L}$, pois apesar de demorar a induzir o peixe aos diferentes estágios de anestesia, provoca uma recuperação rápida (5 minutos).

O tempo de recuperação do tambaqui anestesiado com mentol por 10 minutos ( \pm 11 minutos) é maior que o de peixes da mesma espécie anestesiados com eugenol $( \pm 8$ minutos) (Roubach et al., 2002) e benzocaína ( \pm 5 minutos) (Gomes et al., 2001). O tempo de recuperação de peixes anestesiados com mentol é maior do que o ideal (5 minutos), estando no limite da faixa considerada crítica ( $>10$ minutos) por Marking \& Meyer (1985). Este padrão de recuperação de juvenis já havia sido observado com o eugenol após a anestesia do salmão do atlântico (Salmo salar) (Iversen et al., 2003) e pacu vermelho (Piaractus brachypomus) (Sladky et al., 2001).

A glicose sangüínea é significativamente menor nos peixes expostos a $100 \mathrm{mg} / \mathrm{L}$, do que aos expostos a $200 \mathrm{e}$ $250 \mathrm{mg} / \mathrm{L}$ (Tabela 2). A glicose dos peixes expostos a 150 $\mathrm{mg} / \mathrm{L}$ não difere das demais concentrações testadas. Os valores de glicose mostram que peixes expostos à concentração $150 \mathrm{mg} / \mathrm{L}$, apresentam valores $(73,40 \mathrm{mg} / \mathrm{dL})$ próximos dos basais desta espécie $70 \mathrm{mg} / \mathrm{dL}$ (Gomes et al., 2003). Este resultado é semelhante aos obtidos por Gomes et al. (2001) com $100 \mathrm{mg} / \mathrm{L}$ de benzocaina, e Roubach et al. (2002), com $66 \mathrm{mg} / \mathrm{L}$ de eugenol. Este resultado sugere o mentol como alternativa à benzocaína e ao eugenol durante o manejo do tambaqui.
Os valores plasmáticos $\mathrm{de}^{\mathrm{Na}^{+}}$em peixes anestesiados com mentol não apresentaram diferenças significativas (Tabela 2), mostrando não haver um distúrbio eletrolítico deste íon. Por outro lado, o $\mathrm{K}^{+}$plasmático sofreu aumento significativo nos peixes anestesiados em concentrações superiores a $50 \mathrm{mg} / \mathrm{L}$ (Tabela 2). $\mathrm{O} \mathrm{K}^{+}$é um íon intracelular e seu aumento no plasma significa um rompimento das células (Kirschner, 1991). Este rompimento provavelmente ocorreu nos peixes anestesiados com mentol, pois houve aumento de até três vezes nos valores do $\mathrm{K}^{+}$. Brinn (1999) observou que o tambaqui também apresenta um rompimento celular quando exposto a altas concentrações de cloreto de sódio.

O tempo de recuperação para juvenis expostos a $150 \mathrm{mg}$ L foi de $12,00 \pm 5,44,12,85 \pm 3,61$ e $17,75 \pm 6,41$ minutos para 10,20 e 30 minutos de anestesia, respectivamente. O tempo de recuperação independe do tempo de anestesia até o limite testado de 30 minutos. Também não há mortalidade entre os peixes anestesiados por até 30 minutos. Este resultado é semelhante ao obtido por Keene et al. (1998) para anestesia de truta arco-íris (Oncorbynchus mykiss) com óleo de cravo (derivado do eugenol) por até 20 minutos de duração e diferente do obtido com bagre do canal (Ictalurus punctatus) que apresenta mortalidade após 20 minutos de exposição à concentração ideal de óleo de cravo (Watertrast, 1999). Este resultado reforça a hipótese de que o mentol é um anestésico seguro para o tambaqui.

Não houve diferença no tempo de indução e recuperação entre juvenis e peixes maiores (Figura 1). Este resultado é o inverso ao esperado, pois segundo Roubach et al. (2002) a superfície de absorção é maior nos peixes menores, desta forma, o peixe menor absorve mais anestésicos do que os peixes maiores e conseqüentemente seu tempo de indução a anestesia é mais rápido e sua recuperação mais lenta. Por outro lado, este resultado é favorável, pois se pode recomendar o uso de mentol para a espécie como um todo.

Fazendo uma comparação entre os anestésicos testados para o tambaqui 
(Tabela 3), observa-se que o mentol, de forma geral, atende aos mesmos critérios estabelecidos para um bom anestésico por Ross \& Ross (1999) que a benzocaína e o eugenol. Para que o uso do mentol como anestésico seja aprovado pelas autoridades competentes é necessário conhecer o tempo residual desta substância no tecido do peixe.

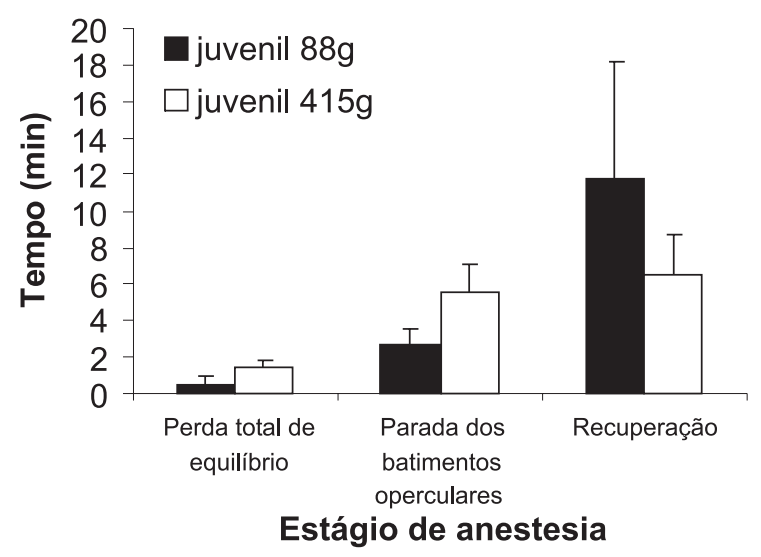

Figura 1 - Tempo para indução aos diferentes estágios de anestesia em juvenis de $88 \mathrm{~g}$ e de $415 \mathrm{~g}$ expostos a $150 \mathrm{mg} / \mathrm{L}$ de mentol. Os estágios de anestesia estão de acordo com os critérios de Stoskopf (1993). Recuperação = recuperação do equilíbrio e natação ativa. As colunas representam os valores médios de 5 peixes de cada tamanho, e as barras o desvio padrão.

\section{CONCLUSÕES}

- O mentol é um anestésico eficaz para o tambaqui;

- A melhor concentração de mentol para uma indução cirúrgica é $150 \mathrm{mg} / \mathrm{L}$. Para uma sedação com finalidade de biometria a melhor concentração é $100 \mathrm{mg} / \mathrm{L}$;

- A exposição do tambaqui ao mentol, em concentração adequada, pode durar até 30 minutos, sem causar mortalidade;

- Exposição à concentração ideal não causa distúrbios fisiológicos de glicose plasmática e no $\mathrm{Na}^{+}$plasmático, porém ocorre um distúrbio no $\mathrm{K}^{+}$plasmático.

\section{AGRADECIMENTOS}

A Pesquisadora Edsandra Campos Chagas, pelo auxílio na análise de íons plasmáticos. Ao Senhor José Pereira de Souza pela coleta e alimentação dos peixes experimentais. Aos alunos de Iniciação científica da Embrapa Amazônia Ocidental: André silva, Lucelle Dantas e Franmir Brandão pela ajuda prestada durante a realização deste trabalho. Trabalho financiado pelos projetos BASA/TANQUE-REDE e TANRE/FINEP.

\section{BIBLIOGRAFIA CITADA}

Brinn, R.P. 1999. Efeitos da salinidade na bomeostase iônica $D L_{50}$ e fluxo iônico do tambaqui, Colossoma macropomum (Cuvier, 1818). Dissertação de Mestrado, Instituto Nacional de Pesquisas da Amazônia/ Universidade Federal do Amazonas, Amazonas. 41pp.

abela 3 - Comparação dos efeitos da benzocaína (Gomes et al., 2001), eugenol (Roubach et al., 2002) e mentol para anestesia de tambaqui. Critérios de acordo com Ross \& Ross (1999).

\begin{tabular}{|c|c|c|c|}
\hline Critério & Benzocaína & Eugenol & Mentol \\
\hline Facilmente administrado & +++ & +++ & +++ \\
\hline $\begin{array}{l}\text { Eficaz em curto } \\
\text { período de exposição }\end{array}$ & +++ & +++ & +++ \\
\hline Eficaz em concentração baixa & +++ & +++ & +++ \\
\hline $\begin{array}{l}\text { Induz ao estado } \\
\text { desejado rapidamente }\end{array}$ & +++ & +++ & +++ \\
\hline Fácil de manter o estado desejado & +++ & +++ & +++ \\
\hline Fácil de inverter o processo & +++ & +++ & +++ \\
\hline Recuperação rápida e tranqüila & +++ & ++ & ++ \\
\hline Margem de segurança & ++ & +++ & +++ \\
\hline Solubilidade na água & + & + & + \\
\hline $\begin{array}{l}\text { Não produzir a hiperatividade } \\
\text { durante a indução }\end{array}$ & + & + & + \\
\hline $\begin{array}{l}\text { Não deve induzir uma } \\
\text { resposta forte de estresse }\end{array}$ & + & ++ & + \\
\hline Segurança ao manipulador & +++ & +++ & +++ \\
\hline $\begin{array}{l}\text { Resíduos insignificantes } \\
\text { no tecido }\end{array}$ & + & +++ & $?$ \\
\hline Baixo tempo residual & + & +++ & $?$ \\
\hline Baixo custo & +++ & +++ & +++ \\
\hline
\end{tabular}

+- razoável; ++- bom; +++ - muito bom; ?- desconhecido.
Gomes, L.C.; Chippari-Gomes, A.R.; Lopes, N.P.; Roubach, R.; AraujoLima, C.A.R. 2001. Efficacy of benzocaine as an anesthetic in juvenile tambaqui Colossoma macropomum. Journal of the World Aquaculture Society, 32:426-431.

Gomes, L.C.; Araujo-Lima, C.A.; Roubach, R.; Urbinati, E.C. 2003. Avaliação dos efeitos sal e da densidade no transporte de tambaqui. Pesquisa Agropecuária Brasileira, 38:283-290.

Keene, J.L.; Noakes, D.L.G.; Moccia, R.D.; Soto, C.G. 1998. The efficacy of clove oil as an anaesthetic for rainbow trout, Oncorbynchus mykiss (Walbaum). Aquaculture Research, 29:89-101.

Kirschner, L.B. 1991. Water and ions. In: Environmental and metabolic animal physiology: comparative animal physiology. $\quad 4^{\mathrm{a}} \mathrm{Ed}$. Cambridge University Press, Cambridge. p. 13-107. 
Hovda, J.; Linley, T.J. 2000. The potential application of hypothermia for anesthesia in adult pacific salmon. North American Journal Aquaculture, 62:67-72.

Iversen, M.; Finstad, B.; Mckinley, R.S.; Eliassen, R.A. 2003. The efficacy of metomidate, clove oil, Aqui-S ${ }^{\mathrm{TM}}$ and Benzoak ${ }^{\circledR}$ as anaesthetic in Atlantic salmon (Salmo salar L.) smolts, and their potentiol stress-reducing capacity. Aquaculture, 221:549-566.

Lorenzo, D.; Paz, D.; Dellacassa, E.; Davies, P.; Vila, R.; Canigueral, S. 2002. Essential oils of Mentha pulegium and Mentha rotundifolia from Uruguay. Brazilian Archives of Biology and Technology, 45:1-6.

Martins, E.R.; Castro, D.M.; Castellani, D.C.; Dias, E. J. 2000. Plantas Medicinais. Ed. UFV, Viçosa, Minas Gerais. 220pp.

Marking, L.L.; Meyer, F.P. 1985. Are better anaesthetics needed in fisheries?, Fisheries 10:2-5.

Mgbenka, B.O.; Ejiofor, E.N. 1998. Effects of extracts of dried leaves of Erythrophleum suaveolens as anesthetics on clariid catfish. Journal of Applied Aquaculture, 8:73-80.

Ross, L.G.; Ross, B. 1999. Anaesthetic and sedative techniques for aquatic animals. Blackwell Science, Oxford. 159pp.

Roubach, R.; Gomes, L.C.; Val, A.L. 2001. Safest level of tricaine methanosulfanate (MSS-222) to induce anesthesia in juveniles of matrinxã (Brycon cephalus). Acta Amazonica, 31:159-163.
Roubach, R.; Gomes, L.C. ; Lourenço, J. N. P.; Fonseca, F.A.L.; Val, A.L. 2002. Efficacy of eugenol as anaesthetic for tambaqui Juvenile (Colossoma macropomum). In: Val, A.L.; Almeida-Val, V.M.F.; McKinley, D. (Eds.) Tropical Fish: News and Reviews. International congress on biology of fishes. Vancouver. p. 93-96.

Roubach, R.; Gomes, L.C. 2001. O uso de anestésicos durante o manejo de peixes. Panorama Aqüicultura, 11:37-40.

Ruppert, E.E.; Barnes, R.D. 1994. Zoologia dos Invertebrados. 6.Ed. Ed. Roca, São Paulo. 1056pp.

Slady, K.K.; Swanson, C.R.; Stoskopf, M.K.; Loomis, M.R.; Lewbart, G.A. 2001. Comparative efficacy of tricaine methanesulfonate and clove oil for use as anesthetics in red pacu (Piaractus brachypomus). American Journal of Veterinary Research, 3:337-342.

Stoskopf, M.1993. Anaesthesia. In: Brown, L. (ed.). Aquaculture for veterinarians: fish busbandry and medicine. Pergamon Veterinary Handbook Series. London. p.161-168.

Waterstrat, P.R. 1999. Induction and recovery from anesthesia in channel catfish Ictalurus punctatus fingerlings exposed to clove oil. Journal of the World Aquaculture Society, 30:250-255.

\section{RECEBIDO EM 09/03/2004 \\ ACEITO EM 22/02/2005}


